Technische

Universität

Berlin

Martin Rohloff, Sevilay Cosgun, Cyriac Massué, Thomas Lunkenbein, Anatoliy Senyshyn, Martin Lerch, Anna Fischer, Malte Behrens

\title{
The role of synthesis conditions for structural defects and lattice strain in beta-TaON and their effect on photo- and photoelectrocatalysis
}

Open Access via institutional repository of Technische Universität Berlin

Document type

Journal article | Published version

(i. e. publisher-created published version, that has been (peer-) reviewed and copyedited; also known as:

Version of Record (VOR), Final Published Version)

This version is available at

https://doi.org/10.14279/depositonce-12536

Citation details

Rohloff, M., Cosgun, S., Massué, C., Lunkenbein, T., Senyshyn, A., Lerch, M., Fischer, A., \& Behrens, M. (2018). The role of synthesis conditions for structural defects and lattice strain in -TaON and their effect on photo- and photoelectrocatalysis. In Zeitschrift für Naturforschung B (Vol. 74, Issue 1, pp. 71-83). Walter de Gruyter GmbH. https://doi.org/10.1515/znb-2018-0171

Terms of use

This work is protected by copyright and/or related rights. You are free to use this work in any way permitted by the copyright and related rights legislation that applies to your usage. For other uses, you must obtain permission from the rights-holder(s). 
Martin Rohloff, Sevilay Cosgun, Cyriac Massué, Thomas Lunkenbein, Anatoliy Senyshyn, Martin Lerch*, Anna Fischer* and Malte Behrens*

\section{The role of synthesis conditions for structural defects and lattice strain in $\beta$-TaON and their effect on photo- and photoelectrocatalysis}

https://doi.org/10.1515/znb-2018-0171

Received August 15, 2018; accepted October 26, 2018

Abstract: The importance of the synthesis conditions on the structural and photocatalytic properties of tantalum oxide nitride was investigated by comparing two variants of phase-pure $\beta$-TaON obtained from application of two different synthesis routes, leading to one unstrained and one heavily anisotropically microstrained $\beta$-TaON as shown by XRD-based Rietveld refinement. HRTEM images reveal the origin of the strain to be lattice defects such as stacking faults. The strained $\beta$-TaON was found to be the clearly less active semiconductor in photochemical and photoelectrochemical water oxidation. The lattice defects are assumed to act as charge carrier traps hindering the photo-generated holes to be displaced to the reaction sites at the surface.

*Corresponding authors: Martin Lerch, Institut für Chemie, Technische Universität Berlin, Straße des 17 Juni 135, 10623 Berlin, Germany, Phone: +49 30314 22603, Fax: +49 3031479656 , E-mail: martin.lerch@tu-berlin.de; Anna Fischer, Institut für anorganische und analytische Chemie, Albert-Ludwigs-Universität Freiburg, Albertstraße 21, 79104 Freiburg, Germany; and Freiburger Zentrum für interaktive Werkstoffe und bioinspirierte Technologien (FIT), Georges-Köhler-Allee 105, 79110 Freiburg, Germany,

Phone: +49 761203 8717, E-mail: anna.fischer@ac.uni-freiburg.de; and Malte Behrens: Fakultät für Chemie und Center for Nanointegration Duisburg-Essen (CENIDE), Universität Duisburg-Essen, Carl-Benz-Straße 199, 47057 Duisburg, Germany, Phone: +49 201183 3684, Fax: +49 201183 3664;

E-mail:malte.behrens@uni-due.de

Martin Rohloff: Freiburger Zentrum für interaktive Werkstoffe und bioinspirierte Technologien (FIT), Georges-Köhler-Allee 105, 79110 Freiburg, Germany; and Institut für anorganische und analytische Chemie, Albert-Ludwigs-Universität Freiburg, Albertstraße 21, 79104 Freiburg, Germany

Sevilay Cosgun: Institut für Chemie, Technische Universität Berlin, Straße des 17 Juni 135, 10623 Berlin, Germany

Cyriac Massué and Thomas Lunkenbein: Fritz-Haber-Institut Berlin, Abteilung Anorganische Chemie, Faradayweg 4, 14195 Berlin, Germany

Anatoliy Senyshyn: Heinz Maier-Leibnitz Zentrum (MLZ), Technische Universität München, Lichtenbergstraße 1, 85748 Garching bei München, Germany
Keywords: photocatalysis; photoelectrochemistry; structural defects; tantalum oxide nitride; water splitting.

Dedicated to: Professor Wolfgang Bensch on the occasion of his $65^{\text {th }}$ birthday.

\section{Introduction}

Tantalum-based nitrides and oxide nitrides have been studied for many years due to their interesting defect chemistry and optical properties. Recently, they have gained a lot of attention due to their potential application in visible light photocatalysis and photoelectrochemistry [1-7]. Amongst a variety of tantalum-based compounds, $\beta$-TaON turned out to be a promising candidate for photochemistry showing potential in "solar water splitting" enabled by a band gap of $2.5 \mathrm{eV}$ allowing a theoretical solar-to-hydrogen efficiency of about $10 \%$ [8], by band edge positions straddling the OER and HER redox potentials which principally enables overall water splitting, and by their stability in aqueous media as well as moderate cost and acceptable earth abundance.

The $\beta$-modification of TaON crystallizes in the monoclinic baddeleyite-type structure (space group $P 2_{1} / c$ ) with an ordered anion arrangement $[1,9]$. Tantalum exhibits a sevenfold coordination by three oxygen atoms and four nitrogen atoms occupying the Wyckoff position $4 e(x, y$, $z)$. Oxygen and nitrogen are located in the same Wyckoff position $4 e$ but with different coordinates [9].

Many efforts and substantial progress have been made regarding the application of $\beta$-TaON for water splitting [10-15]. However, the performance of catalysts prepared in different laboratories is often not readily comparable. Researchers are well aware of the crucial role of the synthesis conditions [16-20], but only seldom a detailed analyses of the real structure of the products including microstructure and defects is provided that may help to understand the observed differences in photochemical activity between different synthesis routes used in different laboratories. 
In this work, we relate the synthesis conditions of $\beta$-TaON with its structural properties and show how the nominally same material can perform drastically differently in photocatalytic and photoelectrochemical water oxidation depending on its defect structure inherited from the synthesis process. Our work thus highlights the importance of the preparation history of semiconductors for photocatalysis or photoelectrochemistry, which turns out to be an extremely important factor when comparing material intrinsic activities for nominally the same compound.

\section{Experimental}

\subsection{TaON powder synthesis}

For all reactions an amount of 300-500 mg crystalline $\mathrm{Ta}_{2} \mathrm{O}_{5}$ (99.99\%, Chempur) was treated with gaseous ammonia in a tightly closed tube furnace equipped with a silica glass tube with a diameter of $55 \mathrm{~mm}$. By means of mass flow controllers (Brooks Instruments) the flow rates of the reaction gases were adjusted. After reaction, a rapid cooling process is important. It should be mentioned that the presence of oxygen plays a crucial role in terms of phase purity. Small amounts of oxygen in the ammonia gas prevent, for example, the formation of $\mathrm{Ta}_{3} \mathrm{~N}_{5}$.

Yellow $\beta$-TaON can be directly synthesized at $900^{\circ} \mathrm{C}$ for $12 \mathrm{~h}$ with flow rates of $10 \mathrm{~L} \cdot \mathrm{h}^{-1}$ ammonia and $0.3 \mathrm{~L} \cdot \mathrm{h}^{-1}$ oxygen. The obtained material is referred to as $\mathbf{1}$ in the following. A green variant of $\beta$-TaON was prepared at $1050^{\circ} \mathrm{C}$ for $12 \mathrm{~h}$ with a heating rate of $400 \mathrm{~K} \mathrm{~min}^{-1}$ and flow rates of $10 \mathrm{~L} \cdot \mathrm{h}^{-1}$ ammonia and $0.3 \mathrm{~L} \cdot \mathrm{h}^{-1}$ oxygen. This material can be converted into yellow $\beta$-TaON by an additional treatment of the green powder at $700^{\circ} \mathrm{C}$ for $15 \mathrm{~min}$ in air yielding 2 . In an attempt to relate the photocatalytic properties rather to differences in the microstructure and not primarily to differences in optical absorption, only the two yellow products were compared catalytically.

\subsection{Characterization}

The products were characterized by both X-ray and neutron powder diffraction. X-ray measurements were carried out using a Panalytical X'Pert PRO diffractometer (Bragg-Brentano geometry, $\mathrm{Cu} K \alpha$ radiation). Neutron scattering investigations were performed at the SPODI diffractometer (Forschungs-Neutronenquelle Heinz-MaierLeibnitz, FRM II, Garching, Germany). For all structural refinements the program package FULLPRoF suite (version 2011) was used, applying a pseudo-Voigt peak profile function [21]. The treatment of the microstructural effects within FuLlProf was done with the Thompson-Cox-Hastings pseudo-Voigt function [22]. Nitrogen and oxygen contents were determined by the hot gas extraction method (LECO TC-300/EF-300). For calibration, $\mathrm{ZrO}_{2}$ and steel were used as standard materials. The accuracy is $\sim 2 \%$ of the presented N/O content. Diffuse reflectance spectra were collected with a JASCO model V670 spectrometer using the non absorbing reflectance standard $\mathrm{MgO}$. The diffuse reflection of the sample is related to the coefficients for absorption $K$ and scattering $S$, via the Kubelka-Munk function $F\left(R_{\infty}\right)$, where $R_{\infty}=R_{\text {sample }} / R_{\text {standard }}$ Eq. (1).

$$
F\left(R_{\infty}\right)=\frac{\left(1-R_{\infty}\right)^{2}}{2 R_{\infty}}=\frac{K}{S}
$$

The optical band gap $E_{\mathrm{g}}$ of the powder samples was obtained using the Tauc Plot Eq. (2), where $A$ is a proportional constant and $h v$ the photon energy. The exponent $n$ denotes the nature of the transition $(n=1 / 2$ for a direct allowed transition, $n=2$ for an indirect allowed transition).

$$
\left[F\left(R_{\infty}\right) h v\right]^{1 / n}=A\left(h v-E_{\mathrm{g}}\right)
$$

The morphology and structure of the TaON powder and electrodes was characterized by scanning electron microscopy (SEM) using a FEG-SEM SU8030 instrument from Hitachi equipped with a $30 \mathrm{~mm}^{2}$ silicon drift detector (SDD) from EDAX for energy dispersive X-ray spectroscopy (EDX). Transmission electron microscopy images were recorded on a Cs-corrected FEI Titan 80-300 device with an acceleration voltage of $300 \mathrm{kV}$.

\subsection{Catalytic testing}

\subsubsection{Chemical water oxidation tests using $\mathrm{AgNO}_{3}$ as a sacrificial agent}

Chemical photocatalytic testing was performed in a batch reactor consisting of a $100 \mathrm{~mL}$ three neck round bottom flask. Pure Ar at a rate of $50 \mathrm{~mL} \mathrm{~min}^{-1}$ was constantly bubbled through $50 \mathrm{~mL}$ of deionized water containing $40 \mathrm{mg}$ catalyst, $85 \mathrm{mg} \mathrm{Ag}\left(\mathrm{NO}_{3}\right)$ and $30 \mathrm{mg} \mathrm{La}_{2} \mathrm{O}_{3}$. The lanthanum oxide acts as a basic buffer to adjust the $\mathrm{pH}$ to 7.5 by scavenging the evolving protons during OER. The gas lines are made of air-tight stainless steel. The out coming gas is bubbled through a concentrated sulphuric acid solution for drying, and analysed using a mass spectrometer (Pfeiffer QMS200 OmniStar quadrupole MS). The reactor 
is covered with aluminum foil and two holes are made in the foil at fixed positions to illuminate the reaction with visible light. The visible light is provided by a double swan neck $150 \mathrm{~W}$ Xe lamp (SCHOTT KL 1500 electronics), equipped with a visible light filter $(\lambda>400 \mathrm{~nm})$. Before illumination, oxygen signal calibration is performed by syringe injection of defined volumes of pure $\mathrm{O}_{2}$ through a septum into the reactor.

\subsubsection{Electrode fabrication}

For photoelectrochemical (PEC) water oxidation experiments electrodes were fabricated out of the powder samples via an electrophoretic deposition method (EPD), described by us previously for $\mathrm{BiVO}_{4}$ powder samples [23]. TaON powders were dispersed in iodine-containing acetone $\left(0.4 \mathrm{mg} \mathrm{mL}^{-1}\right)$ by ultrasonication and were deposited electrophoretically onto a fluorine-doped tin oxide-coated glass slide (FTO, $R=8-12 \Omega$, provided by Sigma Aldrich) in a 2-electrode setup. A potential of $10 \mathrm{~V}$ was applied for 5 min between two parallel substrates at a constant distance of $0.8 \mathrm{~cm}$. After drying in air, the asprepared electrodes were treated five times with $10 \mu \mathrm{L}$ of $10 \mathrm{mM} \mathrm{TaCl}_{5}$ solution in methanol and calcined for $30 \mathrm{~min}$ at $525^{\circ} \mathrm{C}$ in a flow of ammonia $\left(10 \mathrm{~L} \mathrm{~h}^{-1}\right)$, according to a procedure reported by Domen et al. [14].

\subsubsection{Photoelectrochemical measurements}

Photoelectrochemical measurements were carried out in a 3-electrode setup consisting of the TaON working electrode, a counter electrode (Pt wire) and a reversible hydrogen reference electrode (RHE) using $0.1 \mathrm{M}$ potassium phosphate buffer at $\mathrm{pH}$ 7.3. The cobalt phosphate water oxidation catalyst (CoPi) was electrodeposited at a constant potential of $1.7 \mathrm{~V}$ vs. RHE. To ensure a comparable amount of deposited CoPi for all samples, the deposition was stopped after a transferred charge of $60 \mathrm{mC} \mathrm{cm}^{-2}$ was reached. The potential of the working electrode was controlled by a potentiostat (Biologic SP-150). Electrodes were illuminated by a $150 \mathrm{~W}$ Xe lamp in a spectral range from 400 to $700 \mathrm{~nm}$. For $j-V$ measurements the light intensity was adjusted to $100 \mathrm{~mW} \mathrm{~cm}-2$ using a Si diode. For photocurrent transient measurements the light intensity was adjusted to $2 \mathrm{~mW} \mathrm{~cm}^{-2}$ and blue light $(\lambda=440 \mathrm{~nm})$ was used. In all experiments front side illumination was used, i.e. light was incident from the electrolyte side.

\section{Results}

\subsection{Structural characterization}

We compare two different synthesis routes, both leading to bright yellow variants of $\beta$-TaON as described in the experimental section. 1 was obtained in a one-step procedure, while $\mathbf{2}$ was synthesized in a two-step procedure via green $\beta$-TaON as schematically shown in Fig. 1. The two resulting yellow products were structurally characterized using X-ray powder diffraction, which in both cases revealed phase-pure $\beta$-TaON phases.

The structural parameters of $\mathbf{1}$ and $\mathbf{2}$ were refined using the Rietveld method. The refinements are presented in Fig. 2. From the difference plot it is obvious that the crystal structure of $\mathbf{1}$ (Fig. 2a) can be refined without any problems, the experimental data thus being in a very good agreement with the theoretical ones. In clear contrast, the Rietveld refinement of $\mathbf{2}$ led to severe problems (see difference plot (blue) in Fig. 2b), most pronounced for the two major reflections at $2 \theta=29.2^{\circ}$ and $32.7^{\circ}$ corresponding to $h k l$ reflections $\overline{1} 11$ and 111 , respectively.

In Table 1 the lattice parameters and residual values of $\mathbf{1}$ are compared with those of $\mathbf{2}$. Only slight yet significant differences can be observed regarding the lattice parameters. The parameter $S$ providing information about the quality of the refinement is 1.17 for $\mathbf{1}$ and 2.16 for $\mathbf{2}$, clearly indicating the poor fit quality of the latter.

Additionally, the parameters of the intermediate green TaON precursor phase of $\mathbf{2}$, which also crystallizes in the baddeleyite-type structure, were refined. The $\mathrm{X}$-ray powder diffraction diagram with the results of the Rietveld refinement is shown in Fig. 3. It can be seen that the refinement quality is very good. Comparing the structural parameters and residual values of green $\mathrm{TaON}$ with those of $\mathbf{1}$, again slight but significant deviations can be

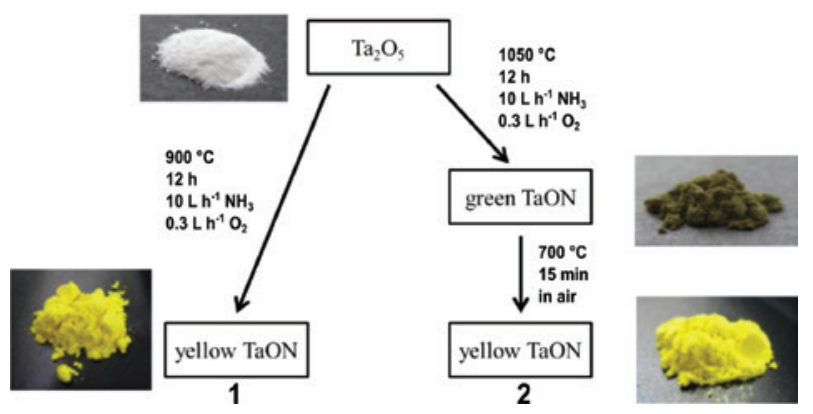

Fig. 1: Scheme of the two different synthesis routes of yellow $\beta$-TaON starting from $\mathrm{Ta}_{2} \mathrm{O}_{5}$. Left: One-step synthesis of the yellow $\mathrm{TaON}$, with the product labelled as 1. Right: Two-step synthesis of the yellow TaON, with the product labelled as 2 . 

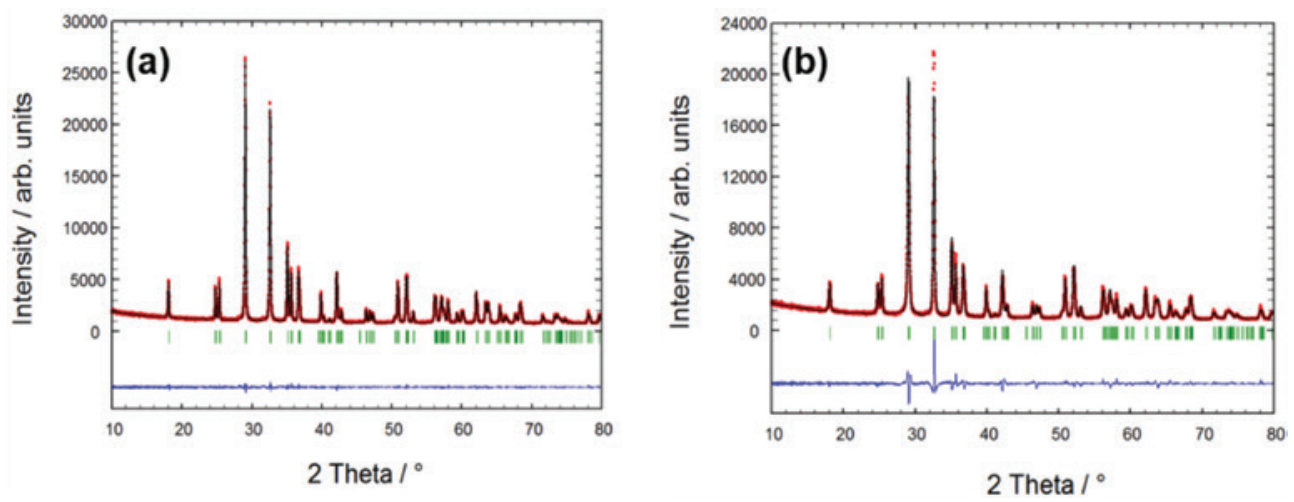

Fig. 2: (a) X-ray powder diffraction diagram of yellow TaON 1 with the results of the Rietveld refinement. (b) X-ray powder diffraction diagram of yellow TaON 2 with the results of the Rietveld refinement. Blue line below: difference between the observed and calculated data. In 2 discrepancies are mainly present at $2 \theta=29.2^{\circ}$ and $32.7^{\circ}$.

Table 1: Lattice parameters and residual values from the Rietveld refinement of 1 (Fig. 2(a)) and 2 (Fig. 2(b)).

\begin{tabular}{|c|c|c|}
\hline & 1 (a) & 2 (b) \\
\hline Structure type & \multicolumn{2}{|c|}{ Baddeleyite } \\
\hline Crystal system & \multicolumn{2}{|c|}{ Monoclinic } \\
\hline Space group & \multicolumn{2}{|c|}{$P 2_{1} / c($ No.14) } \\
\hline Diffractometer & \multicolumn{2}{|c|}{ Panalytical X'Pert PRO } \\
\hline Wavelengths $\lambda / \mathrm{pm}$ & \multicolumn{2}{|c|}{$154.06 ; 154.44$} \\
\hline $2 \theta$ range $/$ deg & \multicolumn{2}{|c|}{$5-80$} \\
\hline $\mathrm{Z}$ & \multicolumn{2}{|c|}{4} \\
\hline$a / \mathrm{pm}$ & 496.856(11) & 496.62(3) \\
\hline$b / \mathrm{pm}$ & $503.664(11)$ & $503.37(3)$ \\
\hline$c / p m$ & $518.444(12)$ & $518.23(3)$ \\
\hline$\beta /$ deg & $90.6043(6)$ & $90.606(2)$ \\
\hline $\mathrm{R}_{\text {Bragg }} / \%$ & 1.18 & 2.80 \\
\hline$R_{\mathrm{wp}} / \%$ & 3.12 & 5.34 \\
\hline$R_{\text {exp }} / \%$ & 2.61 & 2.47 \\
\hline$S$ & 1.20 & 2.16 \\
\hline
\end{tabular}

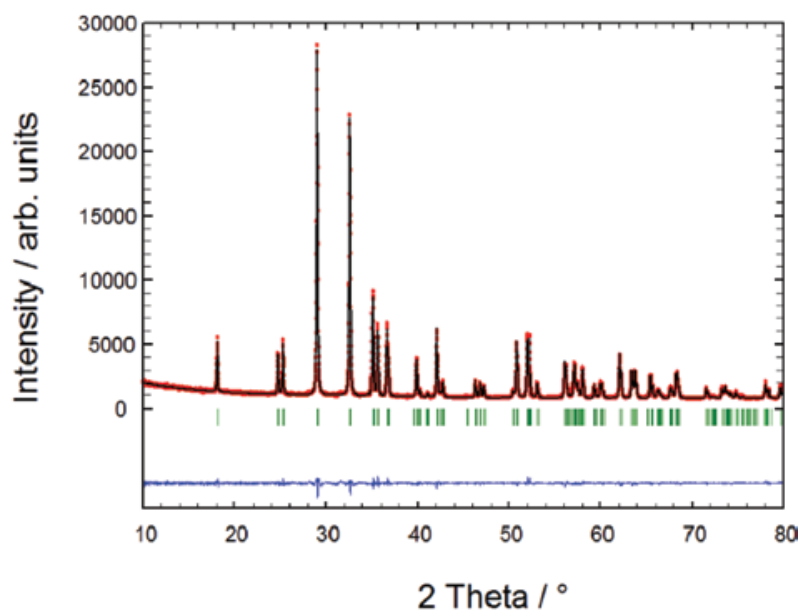

Fig. 3: X-ray powder diffraction diagram of green TaON with the results of the Rietveld refinement. Blue line below: difference between the observed and calculated data. observed, as shown in Tables 2-4. This points to the fact that the severe refinement problems for $\mathbf{2}$ are due to the re-heating process in air.

A possible explanation for the severe refinement problems in the case of $\mathbf{2}$ may be a deviation of the commonly observed ordering scheme of the anions. Different N/O arrangements cannot be distinguished by X-ray diffraction methods, but such anion disorder may lead to local

Table 2: Lattice parameters and residual values from the Rietveld refinement of yellow (1) and green $\mathrm{TaON}$.

\begin{tabular}{|c|c|c|}
\hline & Yellow TaON (1) & Green TaON \\
\hline Structure type & \multicolumn{2}{|c|}{ Baddeleyite } \\
\hline Crystal system & \multicolumn{2}{|c|}{ Monoclinic } \\
\hline Space group & \multicolumn{2}{|c|}{$P 2_{1} / c($ No.14) } \\
\hline Diffractometer & \multicolumn{2}{|c|}{ Panalytical X'Pert PRO } \\
\hline Wavelengths $\lambda / \mathrm{pm}$ & \multicolumn{2}{|c|}{$154.06 ; 154.44$} \\
\hline $2 \theta$ range $/$ deg & \multicolumn{2}{|c|}{$5-80$} \\
\hline Z & \multicolumn{2}{|c|}{4} \\
\hline$a / \mathrm{pm}$ & 496.856(11) & $496.577(8)$ \\
\hline$b / \mathrm{pm}$ & $503.664(11)$ & $503.594(8)$ \\
\hline$c / p m$ & $518.444(12)$ & $518.508(9)$ \\
\hline$\beta / \operatorname{deg}$ & $90.6043(6)$ & $90.6492(5)$ \\
\hline $\mathrm{R}_{\text {Bragg }} / \%$ & 1.18 & 1.05 \\
\hline$R_{\mathrm{wp}} / \%$ & 3.12 & 3.10 \\
\hline$R_{\mathrm{exp}} / \%$ & 2.61 & 2.51 \\
\hline$S$ & 1.20 & 1.24 \\
\hline
\end{tabular}

Table 3: Refined structural parameters of 1 from X-ray diffraction data.

\begin{tabular}{llrrrrr}
\hline Atom & Wyckoff & $\boldsymbol{x}$ & $\boldsymbol{y}$ & $\boldsymbol{z}$ & $\boldsymbol{B}_{\text {iso }}$ & SOF \\
\hline $\mathrm{Ta}$ & $4 e$ & $0.29200(11)$ & $0.04505(9)$ & $0.21531(13)$ & $1.90(3)$ & 1 \\
0 & $4 e$ & $0.0541(16)$ & $0.3294(12)$ & $0.3433(13)$ & $2.4(2)$ & 1 \\
$\mathrm{~N}$ & $4 e$ & $0.448(2)$ & $0.7452(13)$ & $0.481(2)$ & $2.1(2)$ & 1 \\
\hline
\end{tabular}


Table 4: Refined structural parameters of green TaON (precursor of 2) from X-ray diffraction data.

\begin{tabular}{llrrrrr}
\hline Atom & Wyckoff & $\boldsymbol{x}$ & $\boldsymbol{y}$ & $\boldsymbol{z}$ & $\boldsymbol{B}_{\text {iso }}$ & SOF \\
\hline $\mathrm{Ta}$ & $4 e$ & $0.29178(9)$ & $0.04502(8)$ & $0.21445(10)$ & $1.80(2)$ & 1 \\
$\mathrm{O}$ & $4 e$ & $0.0628(13)$ & $0.3283(10)$ & $0.3449(11)$ & $1.84(16)$ & 1 \\
$\mathrm{~N}$ & $4 e$ & $0.4427(15)$ & $0.7517(11)$ & $0.4792(18)$ & $1.5(2)$ & 1 \\
\hline
\end{tabular}

structural distortion significantly influencing the diffraction pattern. In order to exclude an effect of different N/O arrangements, neutron powder diffraction investigations were carried out. It is noted that neutron scattering is the method of choice for such kind of problems because $\mathrm{N}$ and $\mathrm{O}$ strongly differ in their respective coherent scattering length. On the other hand, neutron diffraction is not very sensitive for microstructural peak broadening of highly crystalline samples due to the typically higher contribution of instrumental broadening. Visual inspection of the neutron diffraction patterns and the results of the Rietveld refinements (Fig. $4 a$ and b) clearly indicate that there are no substantial differences in anion ordering between the two yellow products $\mathbf{1}$ and $\mathbf{2}$.

However, small but significant deviations can again be observed in the lattice parameters and the residual values between 1 and $\mathbf{2}$ (see Table 5). Whereas the atom positions are very similar, too small site occupancy factors (SOF) for the anions and significant differences between the samples are detected (see Tables 6 and 7). 1 Exhibits site occupancy factors of 0.958 for oxygen and 0.950 for nitrogen, (expected SOF, $\mathrm{O}=1$ and $\mathrm{N}=1$; hot gas extraction: $7.60 \% \mathrm{O}, 6.66 \% \mathrm{~N}$, resulting in $\mathrm{TaO}_{1.0} \mathrm{~N}_{1.0}$ ). In contrast, in $\mathbf{2}$ slightly more oxygen than nitrogen is present (site occupation factors of 0.986 for oxygen and 0.968 for nitrogen). This under-occupation of nitrogen was also confirmed by the quantitative chemical analysis, but is
Table 5: Refined structural parameters from neutron diffraction data for 1 (Fig. 4a) and 2 (Fig. 4b).

\begin{tabular}{lll}
\hline & 1 (a) & 2 (b) \\
\hline Diffractometer & & SPODI \\
Wavelengths $\lambda / \mathrm{pm}$ & \multicolumn{2}{c}{154.83} \\
$2 \theta$ range $/ \mathrm{deg}$ & $1-150$ \\
$a / \mathrm{pm}$ & & $496.546(8)$ \\
$b / \mathrm{pm}$ & $496.622(7)$ & $503.429(7)$ \\
$c / \mathrm{pm}$ & $503.603(7)$ & $518.108(9)$ \\
$\beta / \mathrm{deg}$ & $518.313(8)$ & $99.5834(10)$ \\
$R_{\text {Bragg }}$ & $99.5708(9)$ & 2.23 \\
$R_{\text {wp }} / \%$ & 2.65 & 3.18 \\
$R_{\text {exp }} / \%$ & 3.27 & 1.69 \\
$S$ & 1.82 & 1.88 \\
\hline
\end{tabular}

Table 6: Refined structural parameters for 1 from neutron diffraction data.

\begin{tabular}{llrrrr}
\hline Atom & Wyckoff & $\boldsymbol{x}$ & $\boldsymbol{y}$ & $\boldsymbol{z}$ & SOF \\
\hline $\mathrm{Ta}$ & $4 e$ & $0.29302(17)$ & $0.0453(2)$ & $0.2153(2)$ & 1.0 \\
$\mathrm{O}$ & $4 e$ & $0.0626(2)$ & $0.3271(2)$ & $0.3453(2)$ & $0.958(4)$ \\
$\mathrm{N}$ & $4 e$ & $0.44380(12)$ & $0.7568(2)$ & $0.48016(12)$ & $0.950(3)$ \\
\hline
\end{tabular}

Table 7: Refined structural parameters for $\mathbf{2}$ from neutron diffraction data.

\begin{tabular}{llrrrr}
\hline Atom & Wyckoff & $\boldsymbol{x}$ & $\boldsymbol{y}$ & $\boldsymbol{z}$ & SOF \\
\hline $\mathrm{Ta}$ & $4 e$ & $0.2926(2)$ & $0.0452(2)$ & $0.2150(2)$ & 1.0 \\
$\mathrm{O}$ & $4 e$ & $0.0623(2)$ & $0.3272(2)$ & $0.3454(2)$ & $0.986(4)$ \\
$\mathrm{N}$ & $4 e$ & $0.44394(13)$ & $0.7567(2)$ & $0.47996(12)$ & $0.968(4)$ \\
\hline
\end{tabular}

in the range of the experimental error: $7.80 \% 0,6.50 \% \mathrm{~N}$, resulting in $\mathrm{TaO}_{1.03} \mathrm{~N}_{0.98}$. Further investigations were performed in order to find out whether the additional dwell
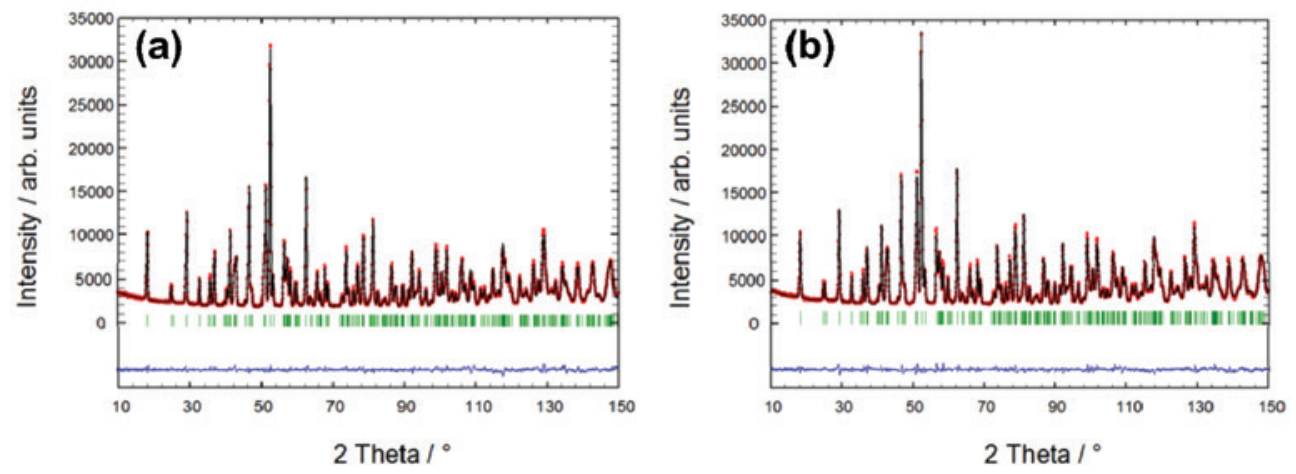

Fig. 4: (a) Neutron powder diffraction diagram of 1 with results of the Rietveld refinement. (b) Neutron powder diffraction diagram of 2 with results of the Rietveld refinement. 
time during the final heating $\mathbf{2}$ in air has an influence on the crystal structure. It was found that a dwell time of up to $20 \mathrm{~min}$ did not afford additional products according to the XRD pattern. However, after 20 min the treatment led to the formation of $\mathrm{Ta}_{2} \mathrm{O}_{5}$ as an additional phase. This observation is in agreement with a roasting-type reaction during air treatment and can explain the higher $\mathrm{O} / \mathrm{N}$ ratio in $\mathbf{2}$ even if no decomposition of the oxide nitride phase has taken place.

Having excluded substantial differences in anion ordering between $\mathbf{1}$ and $\mathbf{2}$ by neutron diffraction experiments, the reflection profiles in the XRD pattern of $\mathbf{2}$ were inspected to find an explanation for the poor fit quality. In Table 8 the full width at half maximum (FWHM), the integral intensity and the ratio of the integral intensity of the two major reflections at $2 \theta=29.2^{\circ}$ and $32.7^{\circ}$ are listed. It is apparent that the FWHM of the two reflections $2 \theta=29.2^{\circ}$ and $32.7^{\circ}$ of $\mathbf{1}$ exhibit similar values. In contrast, the first major reflection at $2 \theta=29.2^{\circ}$ in $\mathbf{2}$ is significantly broader $\left(0.256^{\circ}\right)$. As the ratio of the integral intensity of the major reflections shows hardly any difference for both samples, large differences in the average periodic structure can be excluded. Rather anisotropic reflection broadening, also observed for reflections at higher angles, seems be to the reason for the poor refinement of $\mathbf{2}$.

Usually, the origin of such type of reflection broadening is the presence of structural defects like dislocations, stacking faults, anti-phase domains, micro strains and small anisotropic crystallite size [24]. As confirmed by electron microscopy, both $\mathbf{1}$ and $\mathbf{2}$ show macro crystalline domains (see below), which excludes low crystallite size as the major source of peak broadening. Thus we concentrated our discussion on anisotropic strain broadening. Within the Rietveld refinement, it is possible to model $h k l$ dependent strain broadening which is also known from the equation defined by Warren [25]. It is important to note that the FULLPROF program performs only a phenomenological treatment of reflection broadening in terms of strains due to structural defects. The treatment of microstructural effects takes advantages of the convolution properties of the Voigt

Table 8: FWHMs, integral intensities and ratio of integral intensity for $\mathbf{1}$ and $\mathbf{2}$ (determined applying the pseudo-Voigt profile function).

\begin{tabular}{|c|c|c|c|c|}
\hline & \multicolumn{3}{|c|}{1} & \\
\hline $2 \theta /$ deg & 29.2 & 32.7 & 29.2 & 32.7 \\
\hline FWHM/deg & 0.194 & 0.190 & 0.256 & 0.197 \\
\hline Integral intensity & 4324 & 3705 & 6116 & 5183 \\
\hline Ratio of integral intensity & \multicolumn{2}{|c|}{$1: 0.86$} & \multicolumn{2}{|c|}{$1: 0.85$} \\
\hline
\end{tabular}

function. Within the FULLPROF program the Voigt function is approximated by a pseudo-Voigt function using the Thompson-Cox-Hastings formulae [22]. The anisotropic strain broadening is modeled using a quartic form in the reciprocal space. This corresponds to an interpretation of strains due to correlated static fluctuation between metric parameters [26]. A useful notation in which a phenomenological Lorentzian contribution, the parameter $\xi$, is included into the micro strains was proposed by Stephens [27]. Based on the crystal system there are restrictions on the allowed anisotropic broadening terms. In the case of a monoclinic crystal system nine anisotropic strain parameters $S_{\mathrm{hkl}}$ can be determined.

Interestingly, after applying this anisotropic strain broadening model, a considerable improvement of the refinement quality was achieved (see Fig. 5 and Table 9). A comparison of the refinements with and without the anisotropic strain model shows no significant differences in the lattice parameters, but a considerable improvement

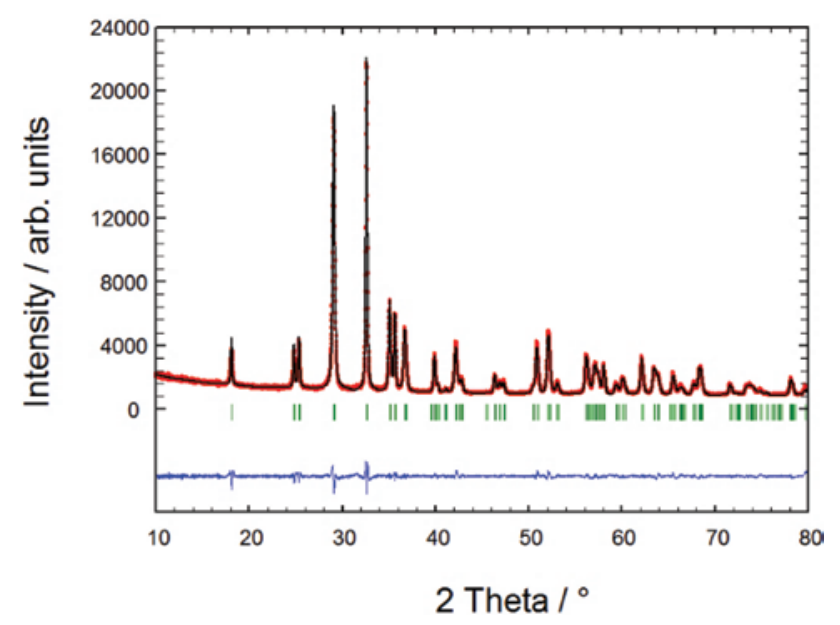

Fig. 5: X-ray powder diffraction diagram of $\mathbf{2}$ after applying an anisotropic strain-broadening model.

Table 9: Lattice parameters and residual values from X-ray diffraction data of $\mathbf{2}$ (with and without microstructural analysis).

\begin{tabular}{lrr}
\hline $\begin{array}{r}\text { 2 Without microstructural } \\
\text { analysis }\end{array}$ & $\begin{array}{r}\text { 2 With microstructural } \\
\text { analysis }\end{array}$ \\
\hline$a / \mathrm{pm}$ & $496.62(3)$ & $496.497(8)$ \\
$b / \mathrm{pm}$ & $503.37(3)$ & $503.306(6)$ \\
$c / \mathrm{pm}$ & $518.23(3)$ & $518.117(8)$ \\
$\beta / \mathrm{deg}$ & $90.6060(15)$ & $90.6070(8)$ \\
$R_{\mathrm{wp}} / \%$ & 5.34 & 4.27 \\
$R_{\exp } / \%$ & 2.47 & 2.47 \\
$S$ & 2.16 & 1.73 \\
\hline
\end{tabular}


Table 10: Refined values of anisotropic strain parameters $S_{\text {hkl }}\left(\times 10^{-4}\right)$ from the microstructural analysis of $\mathbf{2}$.

\begin{tabular}{lr}
\hline Parameter & Value \\
\hline S_400 & $3.93(17)$ \\
S_040 & $0.79(5)$ \\
S_004 & $2.57(11)$ \\
S_220 & $4.1(3)$ \\
S_202 & $7.3(4)$ \\
S_022 & $4.9(3)$ \\
S_121 & $-6.8(5)$ \\
S_301 & $-3.9(4)$ \\
S_103 & $-1.0(4)$ \\
\hline
\end{tabular}

of the residual values. $R_{\mathrm{wp}}$ decreases from 5.34 to 4.27, and therefore so does the value of $S$ from 2.16 to 1.73 . The strain parameters $S_{\text {hkl }}$, which were obtained from the microstructural analysis, are listed in Table 10.
The structural analysis revealed that the major difference between $\beta$-TaON synthesized via the two routes lies in the microstructure of the two resulting $\mathbf{1}$ and $\mathbf{2}$. While only small deviations in the structural parameters including the anion ordering were found, $\mathbf{2}$ is clearly affected by an anisotropic micro strain.

\subsection{TEM investigations}

To further investigate the origin of the anisotropic micro strain in 2, high-resolution TEM investigations were carried out. The corresponding TEM micrographs are represented in Fig. 6. Figure 6a displays a high resolution TEM image of 1 viewed along the crystallographic $c$ axis, for which the atomic structure is almost unaffected. Slight inhomogeneities in the contrast can be explained by thickness differences of the particle. It is noted that

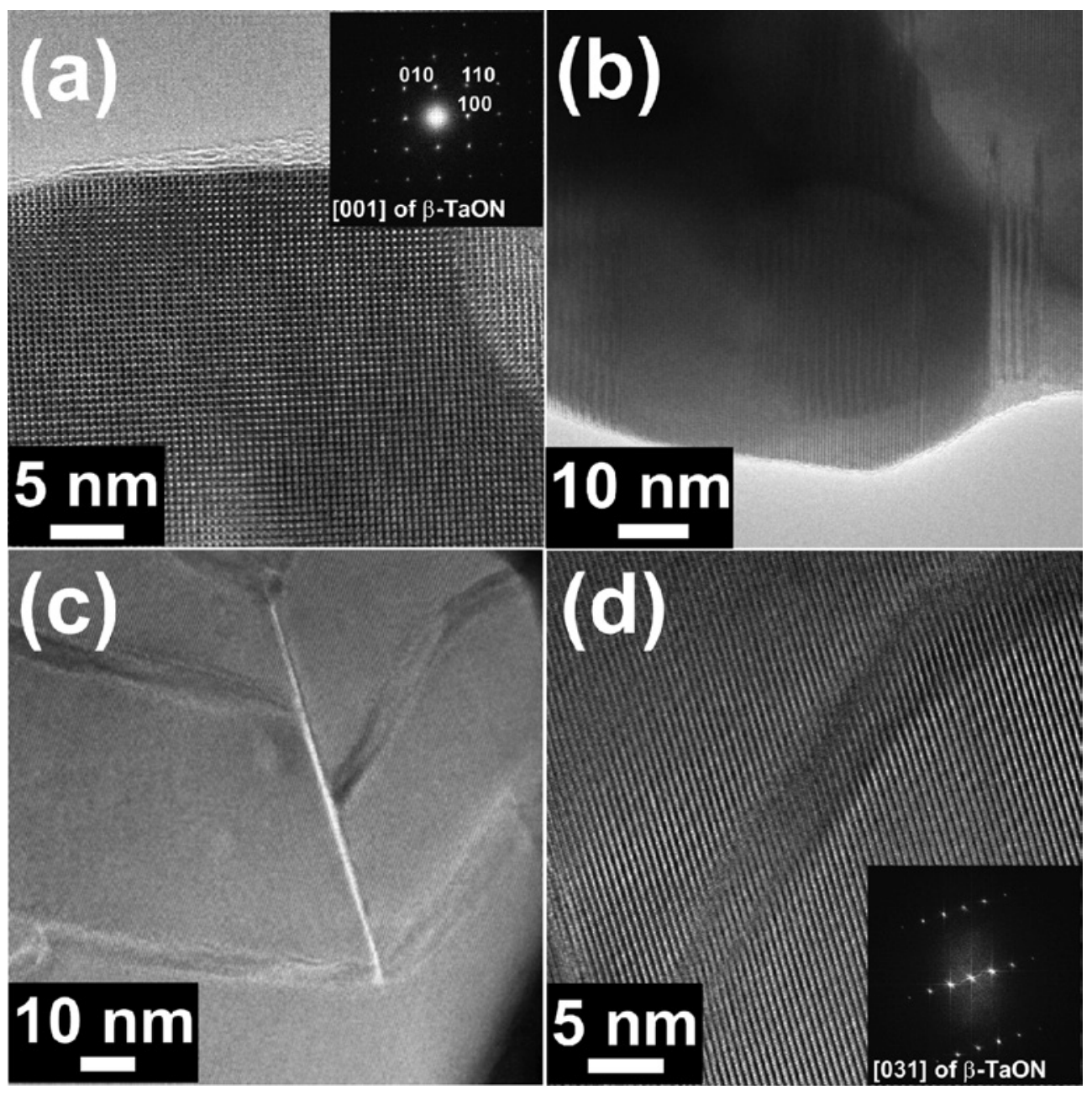

Fig. 6: TEM images of $\beta$-TaON. (a) and (b) display the nanostructure of 1; (c) and (d) show the structure of 2. The insets in (a) and (d) denote Fast Fourier Transformation (FFT) of the corresponding TEM images. 
in agreement with the straightforward refinement of the XRD data, only a small fraction of the investigated particles of $\mathbf{1}$ exhibit defects, such as presented in Fig. 6b. If present, these defects form ordered arrays at the atomic level. As opposed to $\mathbf{1}$, the density of lattice defects in $\mathbf{2}$ is drastically increased. Here, all investigated crystals exhibit lattice defects which are indicated by a varying contrast. Figure $6 \mathrm{c}$ acts as an example, which shows a particle of $\mathbf{2}$ oriented along [031]. The defects penetrate irregularly the atomic structure of $\beta$-TaON. The high resolution TEM image, which is displayed in Fig. 6 d, suggests that the interior structure of the irregular stacking fault is shifted by approximately half of the lattice parameter. Stacking faults are known to cause anisotropic peak profile broadening in the diffraction patterns and thus likely contribute to the observed micro strain in the XRD data.

\subsection{Optical band gaps}

The UV/Vis diffuse reflectance spectra of green $\beta$-TaON and of the two yellow $\beta$-TaON powders (1 and $\mathbf{2}$ ) are shown in Fig. 7. The optical band gaps for all samples were determined based on the Tauc plot of the direct allowed transition. Results are summarized in Table 11. The results for 1 revealed an optical band gap of $2.67 \mathrm{eV}$. In contrast, 2 shows a slightly larger optical band gap of $2.75 \mathrm{eV}$. The increase may be due to more oxygen being present in the lattice of $\mathbf{2}$ making it more oxide-like, which is also in accordance with the $\mathrm{O} / \mathrm{N}$ analysis. For green $\mathrm{TaON}$ a smaller band gap of $2.52 \mathrm{eV}$ was determined. The reason for this difference is most likely due to the presence of $\mathrm{Ta}^{4+}$ after the initial treatment (or $\mathrm{Nb}^{4+}-$ niobium is a known impurity that may be present in traces within the precursor). $\mathrm{Ta}^{4+} / \mathrm{Nb}^{4+}$ in compounds often give the sample a black color. Therefore, the green appearance seems to be due to a mixture of the colors yellow and black. The air treatment of green $\mathrm{TaON}$ then causes a re-oxidation of $\mathrm{Ta} / \mathrm{Nb}$ to their pentavalent state and leads to the disappearance of the black contribution yielding the yellow $\beta$-TaON of 2 .

\subsection{Catalytic properties}

\subsubsection{Chemical water oxidation tests using $\mathrm{AgNO}_{3}$ as a sacrificial agent}

The two yellow samples of $\beta$-TaON, $\mathbf{1}$ and $\mathbf{2}$, were investigated with regard to their photocatalytic performance in the light-driven OER. Oxygen evolution was measured
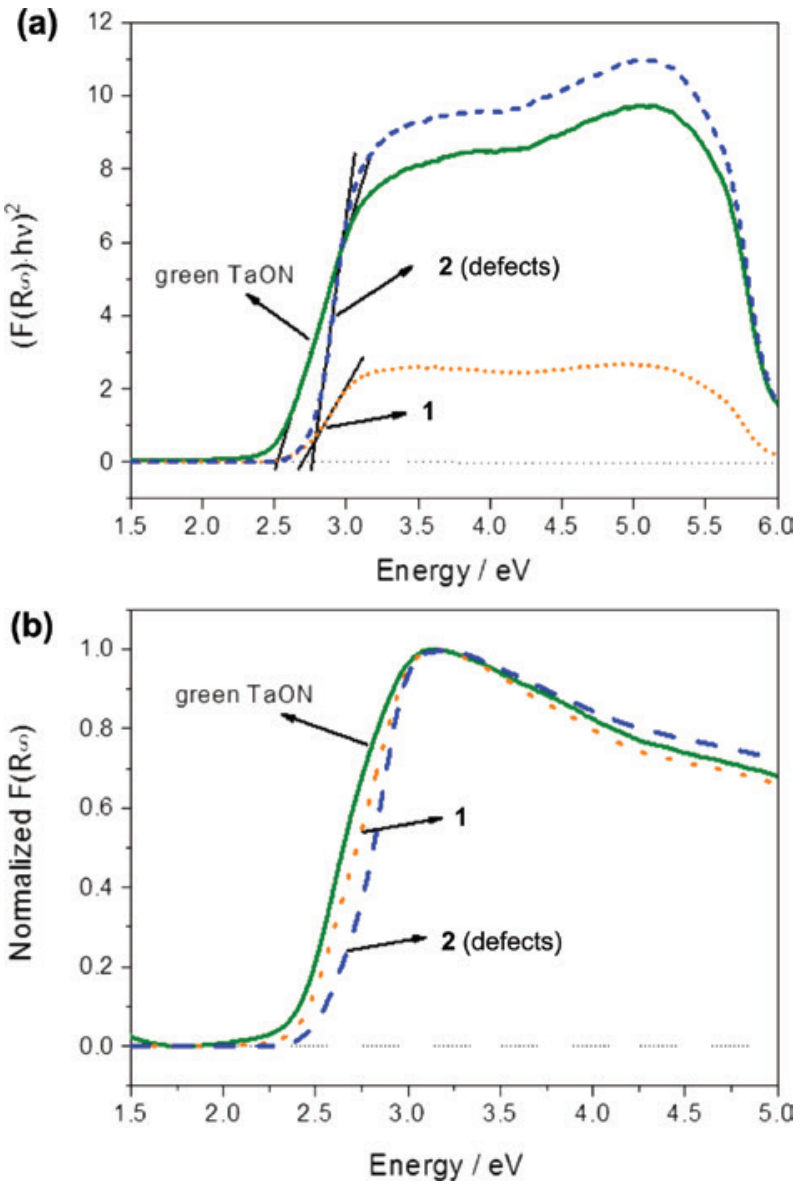

Fig. 7: Diffuse reflectance UV/Vis spectra of 1 and $\mathbf{2}$ (yellow powder $\beta$-TaON) and the green powder. (a) With the corresponding Tauc plot of the direct allowed transition. (b) Normalized Kubelka Munk function. Spectra were recorded at room temperature under ambient conditions.

Table 11: $E_{\mathrm{g}}$ values for all powder samples obtained from UV/Vis reflectance spectroscopy.

\begin{tabular}{lr}
\hline Sample & Band gap (eV) \\
\hline Green TaON & 2.52 \\
1 (yellow TaON) & 2.67 \\
2 (yellow TaON) & 2.75 \\
\hline
\end{tabular}

by irradiating suspensions of $\beta$ - $\mathrm{TaON}$ and $\mathrm{Ag}\left(\mathrm{NO}_{3}\right)$. While the light generated holes serve to oxidize water, $\mathrm{Ag}\left(\mathrm{NO}_{3}\right)$ serves as sacrificial agent for the light induced electrons and gets reduced to Ag. The batch reactor was kept under a constant stream of argon and the outcoming gas was analyzed using mass spectrometry. Figure 8 shows the oxygen evolution measured for $\mathbf{1}$ and $\mathbf{2}$ during visible light irradiation started at $t=0 \mathrm{~min}$. The presence of lattice strain in $\mathbf{2}$ was found to have a sizeable negative 


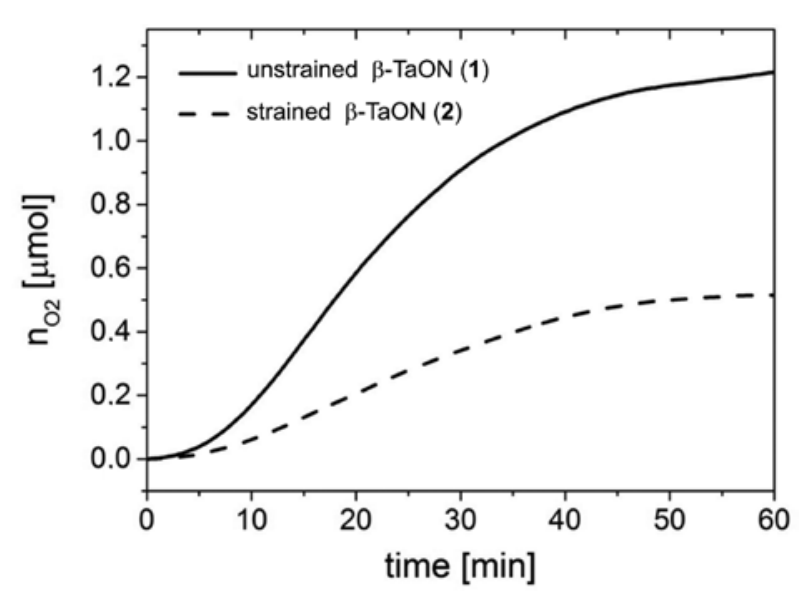

Fig. 8: $\mathrm{O}_{2}$ evolution measured for 1 and $\mathbf{2}$ (unstrained and strained $\beta$-TaON, respectively) under visible light irradiation (light on at $t=0 \mathrm{~min})$ in the presence of $\mathrm{Ag}\left(\mathrm{NO}_{3}\right)$.

effect on the photocatalytic activity. It can be seen that the unstrained 1 produced more than twice as much oxygen than the strained $\mathbf{2}$. The initial rates were determined as the maximum of the first derivatives of the OER curves and used for semi-quantitative comparison. Note that at longer irradiation time, the rates are affected by the formed elemental silver, which interferes with the light absorption and leads to a damping effect on the OER. As a result, the unstrained 1 was shown to initially evolve oxygen at a rate of $2.70 \mu \mathrm{mol} \mathrm{h}^{-1}$, whereas the strained $2 \mathrm{did}$ not exceed an initial OER rate of $1.02 \mu \mathrm{mol} \mathrm{h}^{-1}$. It has been reported that the diffusion length of holes in n-type semiconductors like $\beta$-TaON is rather short, which limits the ability of the holes to reach a reaction center at the photocatalyst's surface to produce oxygen molecules [15]. The various lattice defects observed by HRTEM in $\mathbf{2}$ may act as charge carrier traps and thus as recombination sites further limiting the hole diffusion to the surface offering thereby an explanation for the lower photocatatlytic activity of the strained sample.

\subsubsection{Photoelectrochemistry}

\subsubsection{Electrode fabrication}

To enable photoelectrochemical investigations, electrodes were fabricated out of both yellow $\beta$-TaON powders using an electrophoretic deposition (EPD) method followed by a certain post-treatment, called post-necking in the literature [15]. The synthesis procedure is illustrated schematically in Fig. 9. At every step of the synthesis, the electrodes were investigated by means of electron microscopy and photoelectrochemistry; corresponding images are presented in Fig. 10.

First, powder samples were dispersed in iodine-containing acetone (Fig. 9a). Two glass slides coated with fluorine-doped tin oxide (FTO) were immersed parallel into the dispersion at a constant distance of $0.8 \mathrm{~cm}$. By application of a potential between both FTO substrates TaON particles were deposited electrophoretically (Fig. 9b). As can be seen by HR-SEM (Fig. 10a), the as-prepared electrodes

a)
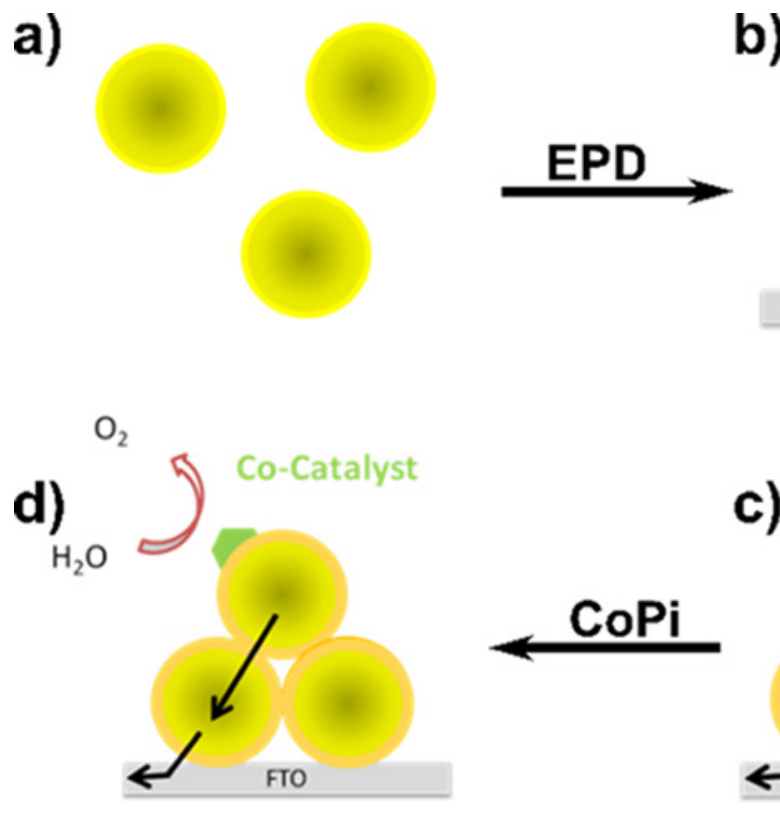

b)
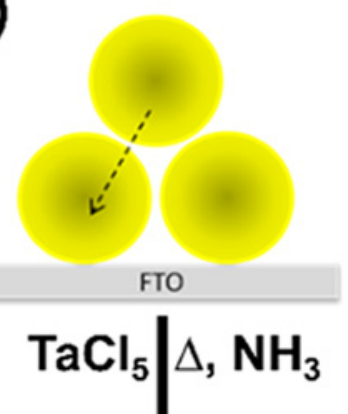

Fig. 9: Synthesis scheme. (a) Sample dispersion in acetone, (b) as-deposited particles at an FTO substrate, (c) post-treated particles with Ta, N, O-containing coating, (d) co-catalyst deposition onto post-treated electrodes. 

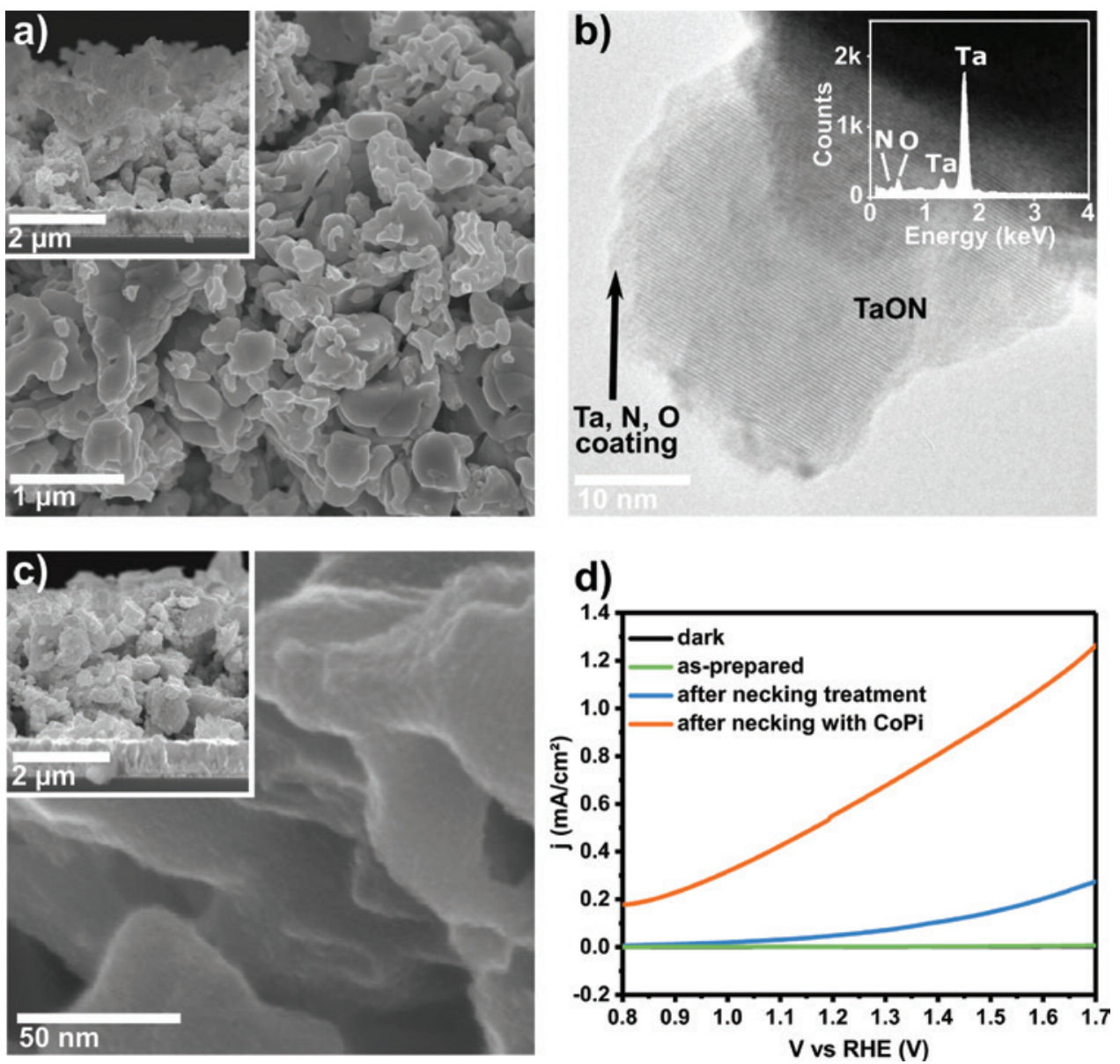

Fig. 10: Screening of the synthesis procedure. (a) SEM top view and cross section images of the as-deposited electrodes. (b) HR-TEM image of a post-treated electrode particle together with an EDX spectrum of the particle coating. (c) Particle surface after CoPi deposition. (d) PEC investigations of unstrained yellow $\beta$-TaON electrodes at every synthesis stage $\left(0.1 \mathrm{M} \mathrm{KPi}, \mathrm{pH}=7.3\right.$, light: $\left.\lambda=400-700 \mathrm{~nm}, 100 \mathrm{~mW} \mathrm{~cm}^{-2}\right)$.

consist of loosely deposited particles which form a particle layer of about $3 \mu \mathrm{m}$ in thickness. To interconnect the deposited particles, a post-treatment comprised of an impregnation with a $\mathrm{TaCl}_{5}$ solution followed by calcination in $\mathrm{NH}_{3}$ atmosphere was applied (Fig. 9c). As a result, a Ta, $\mathrm{N}$ and O-containing coating homogeneously covers and interconnects the deposited particles (in a so-called necking layer, see Fig. 10b). The as post-treated electrodes allow (photo-)electrochemical investigations as well as electrodeposition of a water oxidation co-catalyst (see Fig. 9d). Following the procedure reported by Kanan and Nocera [28], a cobalt phosphate water oxidation cocatalyst (CoPi) was electrodeposited onto the post-treated TaON electrodes. As shown by HR-SEM (Fig. 10c), the $\mathrm{TaON}$ particle surface is significantly roughened after $\mathrm{CoPi}$ electrodeposition which indicates homogeneous surfacecoverage of the TaON particles by the CoPi co-catalyst.

The photoelectrochemical (PEC) performance concerning water oxidation of a TaON photoanode synthesized out of the unstrained yellow $\beta$-TaON powder (1) was investigated at every synthesis stage. Resulting $j-V$ curves are shown in Fig. 10d. As can be seen, for the as-deposited electrode (i.e. after EPD) only very low photocurrents in the range of the dark current were obtained. Improvement of charge transport within the electrode particulate layer could be achieved by connecting the particles with each other with the necking layer, leading to significantly increased photocurrents of $0.08 \mathrm{~mA} \mathrm{~cm}^{-2}$ at $1.23 \mathrm{~V}$ vs. RHE. These photocurrents could be further enhanced by CoPi deposition to $0.53 \mathrm{~mA} \mathrm{~cm}^{-2}$. Obtained photocurrents are not as high as reports on TaON-based photoelectrodes prepared according to this procedure [14]. However, functionality of the electrode synthesis concept is demonstrated.

According to the above-described procedure for photoanode fabrication, electrodes were fabricated out of the unstrained and strained $\beta$-TaON powders and the photoelectrochemical properties regarding water oxidation were investigated. The $j-V$ curves of the unstrained $\beta$-TaON (1) and strained $\beta$-TaON (2) electrodes without surface modification with co-catalyst are shown in 

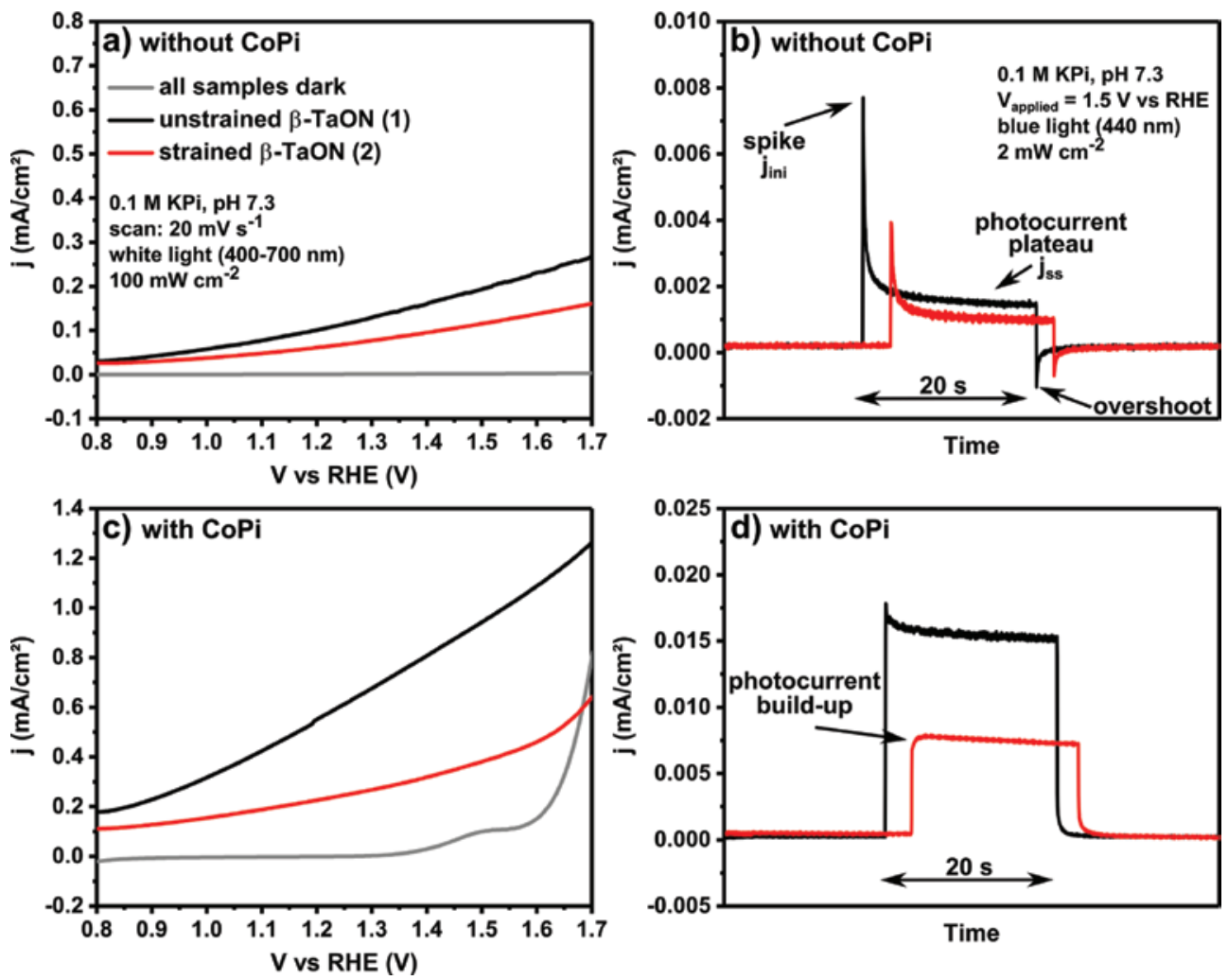

Fig. 11: (a) $j-V$ curves of the unstrained (1) and strained (2) $\beta$-TaON photoanodes. (b) photocurrent transients. (c) $j$ - $V$ curves for the unstrained and strained $\beta$-TaON after CoPi deposition. (d) photocurrent transients after CoPi deposition.

Fig. 11a. Photocurrents accounting for $0.11 \mathrm{~mA} \mathrm{~cm}^{-2}(\mathbf{1})$ and $0.07 \mathrm{~mA} \mathrm{~cm}^{-2}(\mathbf{2})$ at an applied potential of $1.23 \mathrm{~V}$ vs. RHE were obtained, respectively.

To gather information about the reason behind the "limited" photoelectrochemical performance, photocurrent transients were recorded for both samples. Obtained transients were analyzed according to a phenomenological approach developed by Peter et al. [29-32]. As can be seen in Fig. 10b, both samples respond to light on/ off with a characteristic "spike and overshoot" behavior. This response is typical for photoanodes with pronounced electron-hole recombination at the semiconductor surface. When the light is switched on, holes generated in the space charge region are rapidly displaced towards the semiconductor-electrolyte interface. Due to the sluggish kinetics of the water oxidation reaction, holes initially accumulate at the semiconductor-electrolyte interface. In the steady state, the rate of hole arrival is then balanced by the rates of hole transfer towards the electrolyte and the rate of surface electron-hole recombination. As such, the resulting photocurrent transient is the sum of the hole and electron contributions. The instantaneous photocurrent $j_{\text {ini }}$ measured when the illumination is switched on corresponds to a charging or displacement current due to initial movement of photo-generated holes towards the surface. The steady-state photocurrent $j_{\mathrm{ss}}$ corresponds to the flux of holes that are transferred successfully to the electrolyte without undergoing recombination with electrons at the surface. Consequently, the ratio of $j_{\mathrm{ss}}$ and $j_{\text {ini }}$ is a measure for hole transfer efficiency $\eta_{\text {transfer }}$ (see Eq. (3)). Assuming both, hole transfer and surface recombination, to follow are pseudo-first order rate law in terms of surface hole concentration, hole transfer efficiency can be expressed by the corresponding first order rate constants of hole transfer $k_{\text {trans }}$, and hole recombination $k_{\text {rec }}$. The exponential decay of the initial photocurrent $j_{\text {ini }}$ towards the steady state photocurrent $j_{\mathrm{ss}}$ is characterized by the time constants $\left(k_{\text {rec }}+k_{\text {trans }}\right)^{-1}$. The decay can be analyzed and $k_{\text {trans }}$ and $k_{\text {rec }}$ can be separated using Eq. (3). After switching the light off, a cathodic (photo-)current overshoot is observed. The overshoot results from electrons flowing back to the semiconductor surface to recombine with holes (rapid) and with accumulated holes at surface states (slow), a phenomenon called back-electron-hole recombination [33-35].

$$
\eta_{\text {transfer }}=\frac{j_{\text {ss }}}{j_{\text {ini }}}=\frac{k_{\text {trans }}}{k_{\text {rec }}+k_{\text {trans }}}
$$


Table 12: Results of transient analysis for the unstrained and strained $\beta$-TaON photoanodes. Because of the transient shape, application of the analysis model was not applicable to the strained $\beta$-TaON.

\begin{tabular}{lrrr}
\hline & $\begin{array}{r}\text { Hole transfer } \\
\text { efficiency } \boldsymbol{\eta}_{\text {transfer }}\end{array}$ & $\begin{array}{r}\text { Hole transfer } \\
\text { rate } \boldsymbol{k}_{\text {trans }}\left(\mathbf{s}^{-1}\right)\end{array}$ & $\begin{array}{r}\text { Hole recombination } \\
\text { rate } \boldsymbol{k}_{\text {rec }}\left(\mathbf{s}^{-1}\right)\end{array}$ \\
\hline Unstrained $\beta$-TaON & 0.153 & 0.255 & 1.414 \\
Strained $\beta$-TaON & 0.263 & 0.424 & 1.190 \\
Unstrained $\beta$-TaON after CoPi modification & 0.880 & 0.323 & 0.044 \\
Strained $\beta$-TaON after CoPi modification & - & - & - \\
\hline
\end{tabular}

Results of the transient analysis are summarized in Table 12. Hole transfer rates $k_{\text {trans }}$ of $0.255 \mathrm{~s}^{-1}$ and $0.424 \mathrm{~s}^{-1}$ and surface hole-electron recombination rates $k_{\text {rec }}$ of $1.414 \mathrm{~s}^{-1}$ and $1.190 \mathrm{~s}^{-1}$ were determined for the unstrained (1) and strained (2) $\beta$-TaON photoanodes, respectively. Consequently, hole transfer efficiencies $\eta_{\text {transfer }}$ are rather low accounting for 0.153 and 0.263 for the unstrained and strained $\beta$-TaON electrodes, respectively. Thus the transient analysis reveals the PEC performance of both samples, unstrained and strained $\beta$-TaON, to be drastically suffering from surface recombination, even if this effect seems to be more pronounced for the unstrained $\beta$-TaON electrode.

The amorphous cobalt phosphate species "CoPi" was demonstrated in the literature to efficiently reduce surface recombination for instance in combination with $\mathrm{Fe}_{2} \mathrm{O}_{3}$ or $\mathrm{BiVO}_{4}$ photoanodes [34, 36]. For this reason, a thin layer of CoPi was electrodeposited onto the electrodes' surface. Deposition was stopped upon a transferred charge of $60 \mathrm{mC} \mathrm{cm}{ }^{-2}$ to provide a comparable amount of CoPi deposited at both electrodes. $j-V$ curves measured after CoPi functionalization are shown in Fig. 11c. As can be seen, improved PEC performances and photocurrents, respectively, are observed accounting for $0.59 \mathrm{~mA} \mathrm{~cm} \mathrm{~cm}^{-2}$ and $0.24 \mathrm{~mA} \mathrm{~cm}^{-2}$ at an applied potential of $1.23 \mathrm{~V}$ vs. RHE for the unstrained and strained $\beta$-TaON electrodes, respectively. The obtained photocurrents are enhanced by a factor of 5 for the unstrained $\beta$-TaON and by a factor of 3.5 for the strained $\beta$-TaON due to CoPi deposition.

Photocurrent transients for the CoPi-modified electrodes were recorded and analyzed to gain deeper insights into the origin of the unequal improvement of PEC performance. Results are shown in Fig. 11d. As can be seen, due to CoPi deposition the typical spike and overshoot behavior was reduced to a minimum for the unstrained $\beta$-TaON (1). In comparison with the non-CoPi-modified unstrained $\beta$-TaON (1 in Fig. 11b), transient analysis reveals a slightly enhanced hole transfer rate of $0.323 \mathrm{~s}^{-1}$ and a remarkably reduced surface hole recombination rate of $0.044 \mathrm{~s}^{-1}$. Consequently, the hole transfer efficiency $\eta_{\text {transfer }}$ is significantly increased accounting for 0.880 . Contributions of surface hole recombination become negligibly small upon CoPi deposition, hence the PEC performance of the unstrained $\beta$-TaON electrode is drastically improved. The photocurrent overshoot observed when the light was switched off is replaced by a slight tailing or broadening of the transient shape. This usually is attributed to a certain energetic disorder of the material most probably having its origin in the only poorly crystalline post-necking layer formed within the framework of the electrode preparation procedure.

For the CoPi-modified strained $\beta$-TaON (2), a different transient shape is observed. Reduction of surface hole recombination to a minimum due to CoPi deposition results in a rather slow build-up in photocurrent when the light is switched on. This slow rise is usually attributed to the presence of traps within the material, which first need to be filled with photo-generated charge carriers before the steady state photocurrent plateau can be reached [20]. Furthermore, the overshoot is replaced by a tailing of the transient as also observed for the CoPi-modified unstrained $\beta$-TaON (1). In addition, the steady state photocurrent $j_{\mathrm{ss}}$ is significantly smaller for the strained $\beta$-TaON (2) compared with its unstrained counterpart (1). In addition to the transient shape, this further indicates a higher number of recombination sites within the strained $\beta$-TaON leading to lower PEC water oxidation performance.

Analysis of the photocurrent transients reveal the strain defects to act as traps for photo-generated charge carriers and as recombination sites. Hence, the PEC performance of the CoPi-modified strained $\beta$-TaON photoanodes is significantly lower compared to its unstrained counterpart.

\section{Conclusion}

The importance of the synthesis conditions on the structural, photocatalytic and photoelectrochemical properties of tantalum oxide nitride was investigated by comparing two variants of phase-pure $\beta$-TaON obtained from application of two different synthesis routes. One synthesis product was strongly affected by anisotropic microstrain 
as revealed by XRD pattern refinement. HRTEM images have shown that the origin of the strain is due to lattice defects such as stacking faults. These faults were found to be highly detrimental for the photocatalytic and photoelectrochemical oxygen evolution reactions likely because they act as charge carrier traps hindering the photo-generated holes to diffuse to the reaction sites at the surface. This work thus highlights the importance of high crystallinity and defect minimization for semiconductors as photo(electro)catalysts and the need for a detailed investigation of the semiconductors' microstructure in order to fully understand differences in photocatalytic performance between samples of the same compound.

Acknowledgements: The authors would like to thank B. Hahn for performing $\mathrm{O} / \mathrm{N}$ analyses and S. Kühn for UV/ Vis measurements. Financial support from the Deutsche Forschungsgemeinschaft (DFG) within the priority program SPP 1613 (FI 1885/1-2, LE 781/13-2 and BE 4767/2-2) is gratefully acknowledged.

\section{References}

[1] G. Brauer, J. Weidlein, J. Strähle, Z. Anorg. Allg. Chem. 1966, 348, 298.

[2] M. Weishaupt, J. Strähle, Z. Anorg. Allg. Chem. 1977, 429, 261.

[3] C. Zhen, R. Chen, L. Wang, G. Liu, H.-M. Cheng, J. Mater. Chem. A 2016, 4, 2783.

[4] T. Takata, C. Pan, K. Domen, Sci. Tech. Adv. Mater. 2015, 16, 33506.

[5] W. R. Matizamhuka, I. Sigalas, M. Herrmann, Ceram. Int. 2008, 34, 1481.

[6] M. Hara, G. Hitoki, T. Takata, J. N. Kondo, H. Kobayashi, K. Domen, Catal. Today 2003, 78, 555.

[7] W.-J. Chun, A. Ishikawa, H. Fujisawa, T. Takata, J. N. Kondo, M. Hara, M. Kawai, Y. Matsumoto, K. Domen, J. Phys. Chem. B 2003, $107,1798$.

[8] B. Viswanathan, V. Subramanian, J. S. Lee, Materials and Processes for Solar Fuel Production, Springer, New York, NY, 2014.

[9] D. Armytage, B. E. F. Fender, Acta Crystallogr. 1974, B30, 809.
[10] J. Hou, C. Yang, H. Cheng, S. Jiao, O. Takeda, H. Zhu, Energy Environ. Sci. 2014, 7, 3758.

[11] K. Maeda, M. Higashi, D. Lu, R. Abe, K. Domen, J. Am. Chem. Soc. 2010, 132, 5858.

[12] K. Maeda, D. Lu, K. Domen, Chem. Eur. J. 2013, 19, 4986.

[13] M. Higashi, R. Abe, A. Ishikawa, T. Takata, B. Ohtani, K. Domen, Chem. Lett. 2008, 37, 138.

[14] R. Abe, M. Higashi, K. Domen, J. Am. Chem. Soc. 2010, 132, 11828.

[15] M. Higashi, K. Domen, R. Abe, J. Am. Chem. Soc. 2012, 134, 6968.

[16] Y. Sasaki, Z. Tokuyasu, Y. Ono, M. Iwasaki, S. Ito, Adv. Mater. Sci. Eng. 2009, 2009, 1.

[17] M. de Respinis, M. Fravventura, F. F. Abdi, H. Schreuders, T. J. Savenije, W. A. Smith, B. Dam, R. van de Krol, Chem. Mater. 2015, 27, 7091

[18] B. A. Pinaud, A. Vailionis, T. F. Jaramillo, Chem. Mater. 2014, 26, 1576.

[19] T. Jing, Y. Dai, X. Ma, W. Wei, B. Huang, RSC Adv. 2015, 5, 59390.

[20] A. Dabirian, R. van de Krol, Chem. Mater. 2015, 27, 708.

[21] J. Rodríguez-Carvajal, FullPRof2000, A Program for Rietveld Refinement and Pattern Matching Analysis, Satellite Meeting on Powder Diffraction of the $15^{\text {th }}$ International Congress of the IUCr, Toulouse (France) 1990, p. 127.

[22] P. Thompson, D. E. Cox, J. B. Hastings, J. Appl. Crystallogr. $1987,20,79$.

[23] B. Anke, M. Rohloff, M. G. Willinger, W. Hetaba, A. Fischer, M. Lerch, Solid State Sci. 2017, 63, 1.

[24] J. Rodriguez-Carvajal, Physica B 1993, 55, 192.

[25] B. E. Warren, $X$-ray diffraction, Dover, New York, 1990.

[26] J. Rodríguez-Carvajal, M. T. Fernandez-Diaz, J. L. Martinez, J. Phys.: Condens. Matter 1991, 3, 3215.

[27] P. W. Stephens, J. Appl. Crystallogr. 1999, 32, 281.

[28] M. W. Kanan, D. G. Nocera, Science 2008, 321, 1072.

[29] L. M. Abrantes, L. M. Peter, J. Electroanal. Chem. 1983, 150, 593.

[30] L. M. Peter, J. Li, R. Peat, J. Electroanal. Chem. 1984, 165, 29.

[31] L. M. Peter, Chem. Rev. 1990, 90, 753.

[32] L. M. Peter, J. Solid State Electrochem. 2013, 17, 315.

[33] F. Le Formal, S. R. Pendlebury, M. Cornuz, S. D. Tilley, M. Grätzel, J. R. Durrant, J. Am. Chem. Soc. 2014, 136, 2564.

[34] Y. Ma, F. Le Formal, A. Kafizas, S. R. Pendlebury, J. R. Durrant, J. Mater. Chem. A 2015, 3, 20649.

[35] Y. Ma, S. R. Pendlebury, A. Reynal, F. Le Formal, J. R. Durrant, Chem. Sci. 2014, 5, 2964.

[36] M. Barroso, A. J. Cowan, S. R. Pendlebury, M. Grätzel, D. R. Klug, J. R. Durrant, J. Am. Chem. Soc. 2011, 133, 14868. 